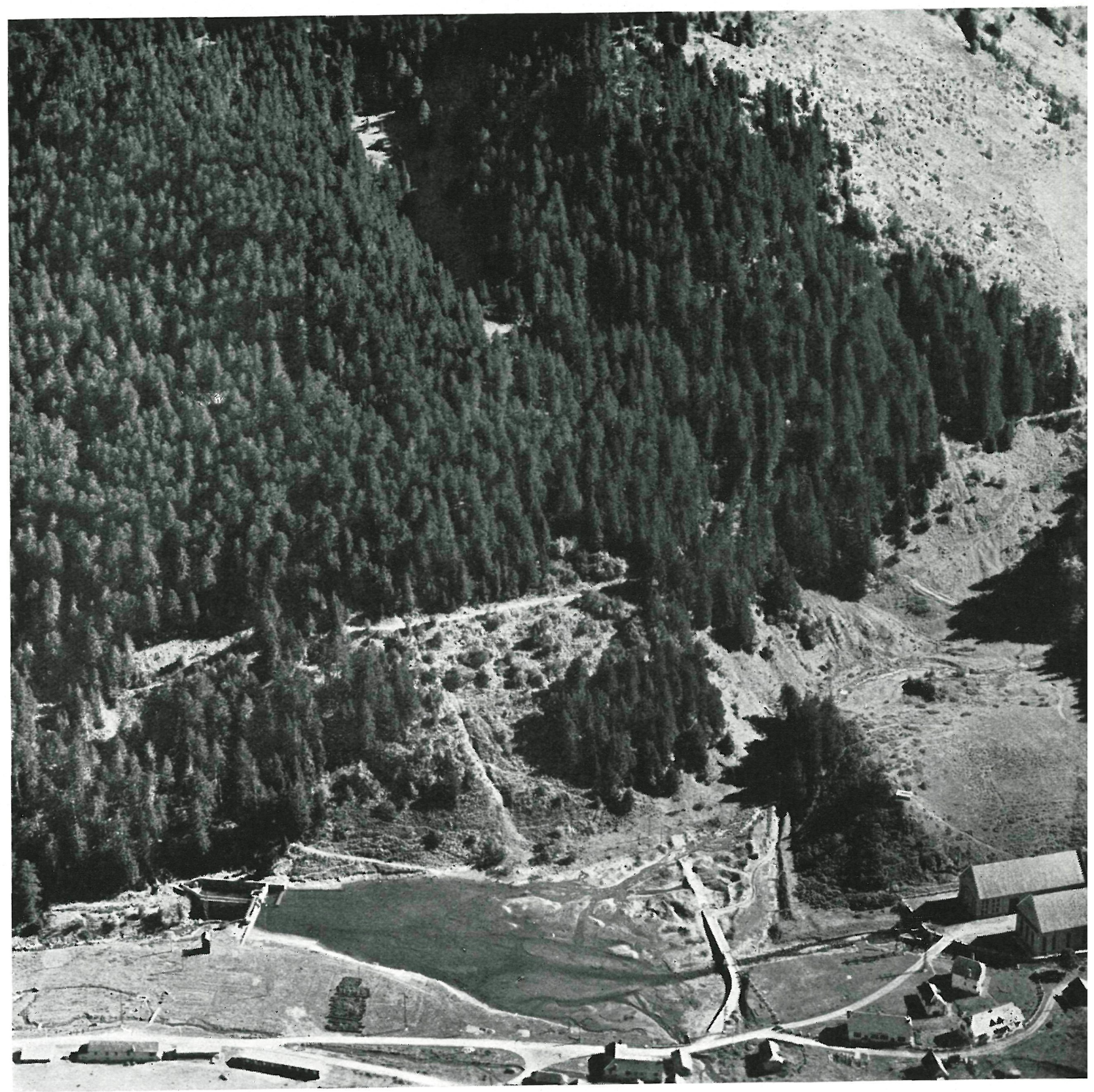

\title{
perforación y revestimiento de galerías
}

$816-6$

consideraciones sobre las dificultades más caracterizadas en esta clase de trabajos

GEORGES VIÉ, ingeniero

S I $\quad$ N $O$ O

En este trabajo se describen algunas de las dificultades que se presentan en la perforación de galerías subterráneas dedicadas a la conducción de agua para su aprovechamiento en una central hidroeléctrica. De todas estas dificultades, las mas importantes son: el agua, su circulacion y la naturaleza de terreno se ha de atravesar en socán del hormigón que las ha de revestir. 


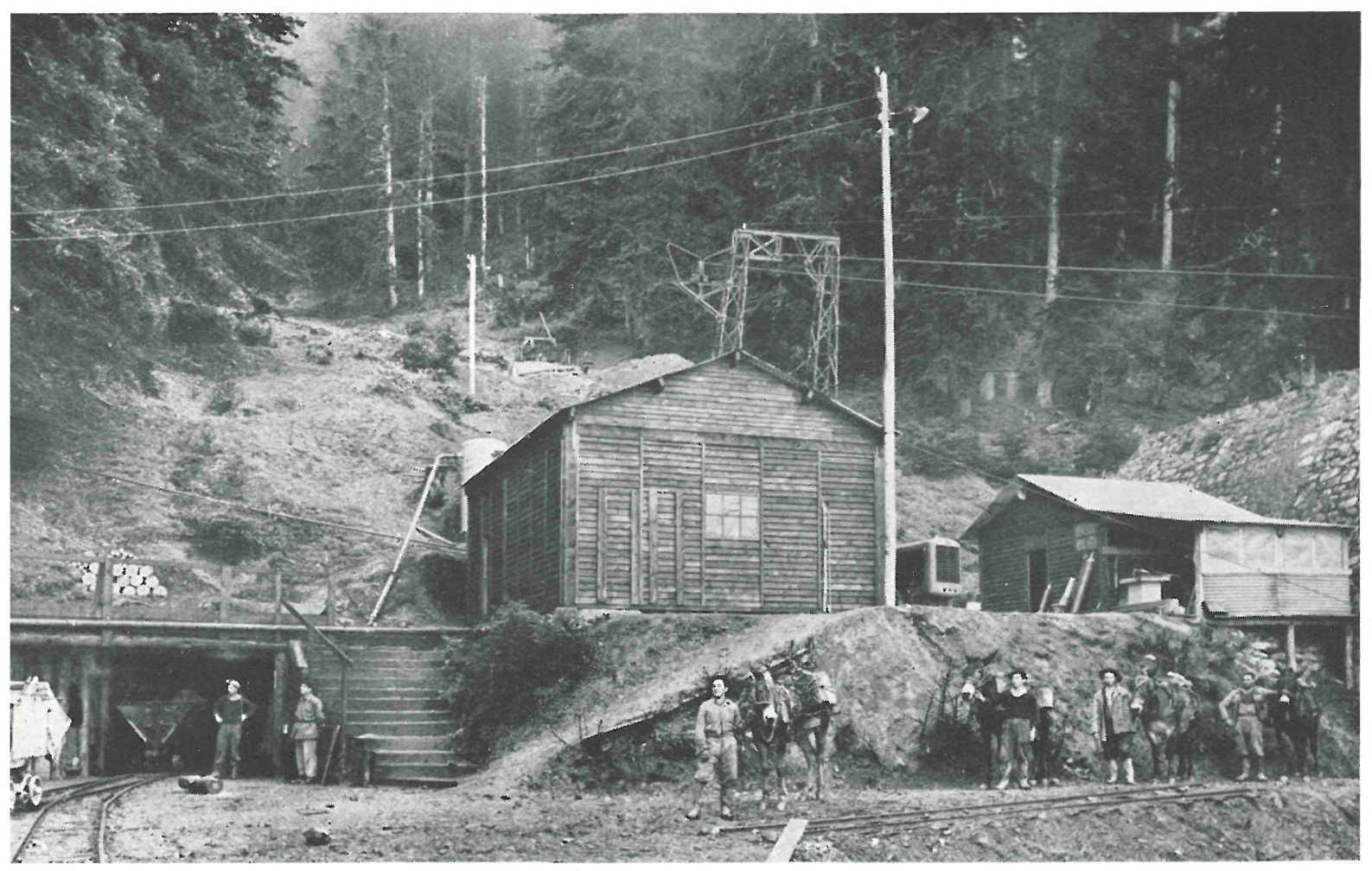

ingeniería

Aspecto de la salida de la galería en su extremidad de aguas abajo.

Ensanchamiento de la guía de cuadros metálicos a una sección mayor, entibada con marcos de madera.

El empuje en el techo sobre los cuadros metálicos fué tan fuerte que tuvieron que apuntalarse.
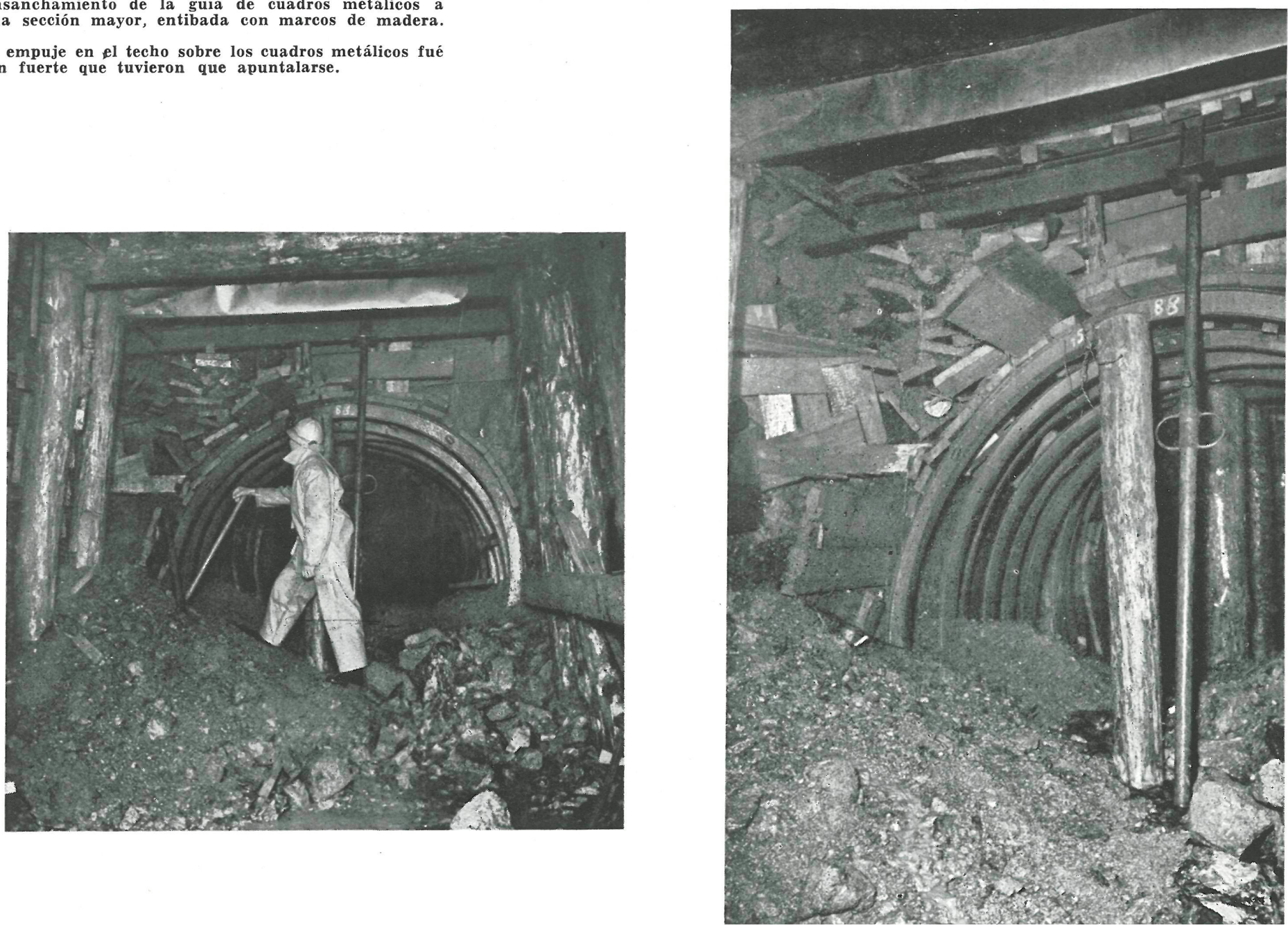


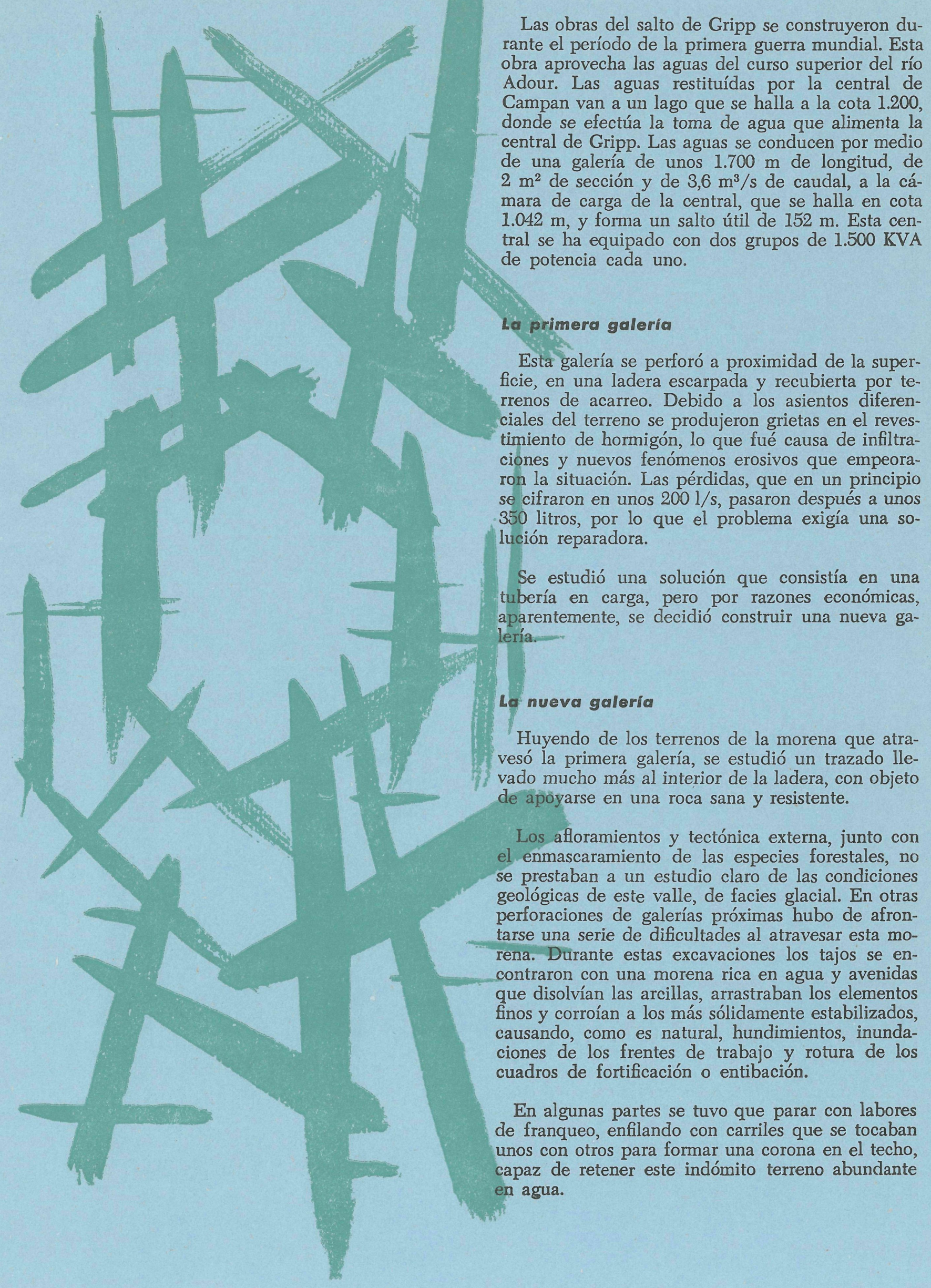


Teniendo en cuenta todo este estado de cosas, se hizo uso de una prospección eléctrica del terreno para tener una idea general de los que debía atravesar el nuevo trazado de la galería, máxime si se procedía a una comprobación práctica por medio de sondeos. La prospección de terrenos por procedimientos eléctricos presenta notables ventajas sobre los sondeos ordinarios, pues la primera alcanza mayores volúmenes de bancos o zonas, mientras que los sondeos, particularmente en zonas de terrenos de acarreos y morenas, como es el caso que nos ocupa, no sólo precisa la posición de un punto único del terreno, sino que puede penetrar en una gran masa rocosa extraña al terreno que la rodea, pararse la perforación en ella y enmascarar los resultados.

Por razones de rapidez y economía los procedimientos geofísicos presentan notables ventajas; sin embargo, tratándose de terrenos tan heterogéneos como los de facies glacial, de acarreo y de abundante circulación hidrológica, se comprenderá fácilmente que los procedimientos geofísicos, aplicados a zonas tan limitadas, en las que además el cambio de posición de los materiales «in situ» tiene una marcada influencia para determinar el trazado más favorable y menos expuesto a encontrarse con sorpresas durante la perforación de la galería, constituye un problema de difícil solución, máxime si tenemos en cuenta la presencia del agua en el subsuelo.

Por todo lo expuesto, se comprenderá que los resultados geológicos obtenidos en la prospección geofísica no coincidieron en la mayoría de los casos con la clase de terreno que atravesó la galería. Durante los trabajos de excavación, los avances del frente se hallaban subordinados en cierto modo a los datos que se adquirían en los sondeos exteriores y los que se realizaban desde el interior de la galería, cuya perforación tuvo que hacer frente a una serie de lisos y dislocaciones locales de gran dificultad e importancia.

Presenta un gran interés dedicar toda la atención posible al estudio previo del terreno y trazado de una galería que ha de atravesar terrenos perturbados tectónicamente, pues es el único medio de poder llegar a conclusiones que, si no absolutas, disminuyan los riesgos de tener que enfrentarse con lisos, avenidas de agua y dificultades de perforación, que siempre encarecen y retardan la obra.

Los terrenos atravesados por la galería son de tipo esquistoso y calizo. Los trabajos empezaron con un solo frente de ataque, que, debido al retraso que dieron lugar las dificultades encontradas, se abrió un segundo tajo en la otra extremidad de la galería, cuando ésta se hallaba ya a unos $1.450 \mathrm{~m}$ del primer socavón. Esta forma de operar no sólo ganó tiempo, sino que, al romper, se pudo organizar el revestimiento partiendo de ambos lados de la galería.

\section{La perforación y sus dificultades}

Las partes de morena atravesadas por la galería se entibaron con cuadros de madera, que se colocaban a razón de dos por día, dando un avance real de 1,80 m por día. En esta zona se encontraron dos fallas importantes que permitieron desaguar el terreno, ya que no se hallaban completamente rellenas.

Los terrenos esquistosos que seguían a los de la morena se pasaron con cuadros metálicos, de $2,30 \mathrm{~m}$ de altura, 2,35 de anchura en el pie y 1,86 en arranque del arco o cabezal. Estos cuadros se arriostraron entre sí por medio de varillas de redondo de $25 \mathrm{~mm}$ de diámetro. El arranque se verificó siguiendo los procedimientos ordinarios, cargando el escombro con palas mecánicas EMCO 21. La plataforma de entrada del socavón de aguas abaijo se alimentaba de materiales y herramientas por medio de un cable aéreo.

En una zona muy acuífera, los cuadros metálicos cedían mucho en corona, por lo que se tuvieron que apuntalar y volver a ensanchar la sección, entibando esta vez con cuadros trapezoidales de madera. En esta zona el agua aparecía a presión, no ya en el techo, sino en solera también, teniendo que hacer frente a labores difíciles y penosas con una sucesión casi continua de hundimientos y obligando a proceder al hormigonado de esta zona inmediatamente después de haberla pasado. Estas dificultades se hicieron sentir en otra parte del trazado que atraviesa esquistos pizarrosos y calizas, donde también hubo que hormigonar rápidamente para mayor seguridad.

A excepción de unos $1.100 \mathrm{~m}$ de galería, perforados dentro de un régimen ordinario, el resto ha presentado siempre una dificultad más o menos apreciable, y en un hundimiento se sacaron hasta $470 \mathrm{~m}^{3}$ de escombro.

\section{Revestimiento}

Los encofrados utilizados para el revestimiento de las galerías son del tipo telescópico, de la casa Blaw-Knox. Se emplearon dos modelos, uno para un diámetro de $1,70 \mathrm{~m}$ y el otro de $2 \mathrm{~m}$. Los espesores del revestimiento varían, según las condiciones de la galería, de 0,15 a $0,30 \mathrm{~m}$. En algunas partes el revestimiento se ha armado.

El hormigón, de áridos de caliza, tenía la composición media siguiente:

$350 \mathrm{~kg}$ de cemento portland.

1.015 a $1.050 \mathrm{~kg}$ de arena de 0/10.

820 a $995 \mathrm{~kg}$ de gravilla 6/18.

240 a 250 litros de agua por metro cúbico en obra. 


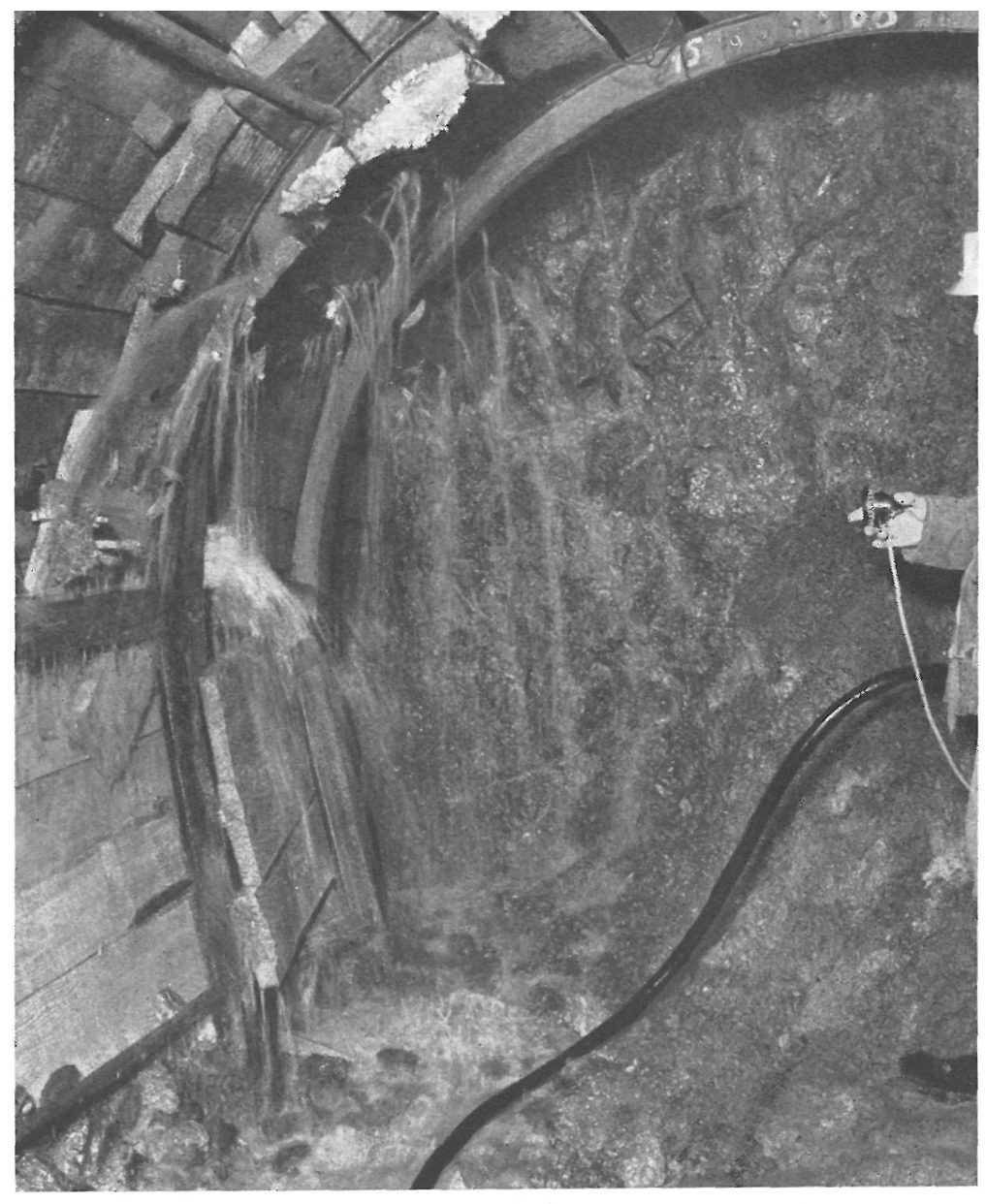

Una de las grandes dificultades de la perforación consistió en la presencia de fuertes avenidas de agua.

Uno de los hundimientos de la zona correspondiente al paso de la morena, agravado por pondiente al paso de la mo

La proporción de agua de la mezcla se aumentó debido a la formación de una harina abundante originada por la friabilidad de la caliza de los áridos. Para una arena determinada el agua era el $12 \%$.

En el tajo correspondiente a aguas abajo de la galería se logró un avance medio semanal de unos $52 \mathrm{~m}$, que se vibraron convenientemente.

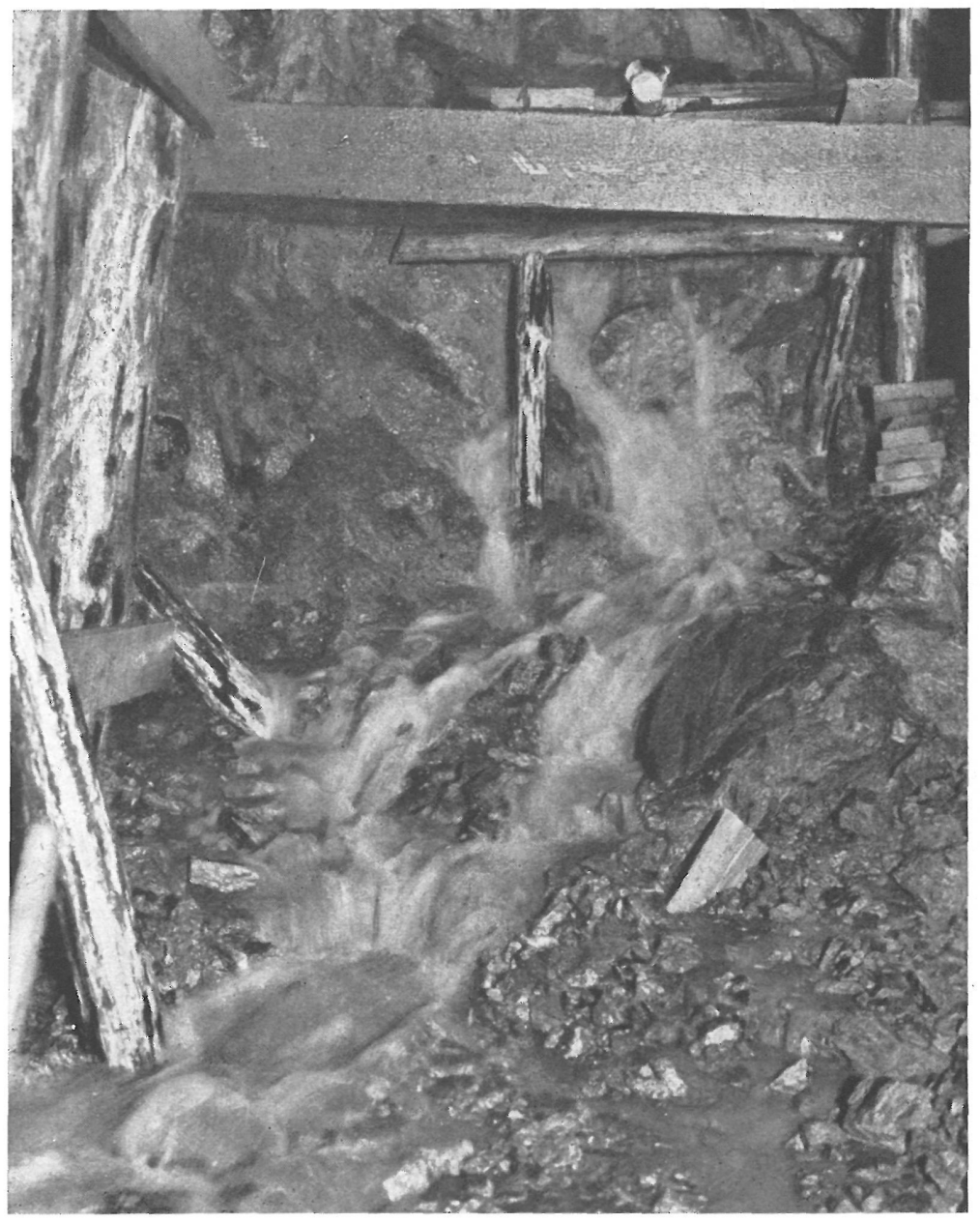

\section{Algunas observaciones}

Al principio se hormigonaba en el exterior, pero cuando el transporte necesitaba un gran recorrido, se presentaba una separación en el hormigón, lo que obligó a que éste se preparase preferentemente en el interior.

El exceso de agua debido a la formación de harinas antes apuntada, fué causa de una fisuración, que se manifestó con mayor intensidad en las zonas armadas que en las de hormigón en masa.

Finalmente, terminado el revestimiento, se procedió a la ejecución de un programa previsto para las inyecciones, encargadas de rellenar huecos, las cuales, en este caso, eran del tipo de un centímetro de altura en las zonas normales y, naturalmente, bastante mayores en las partes entibadas.

J. J. U. 

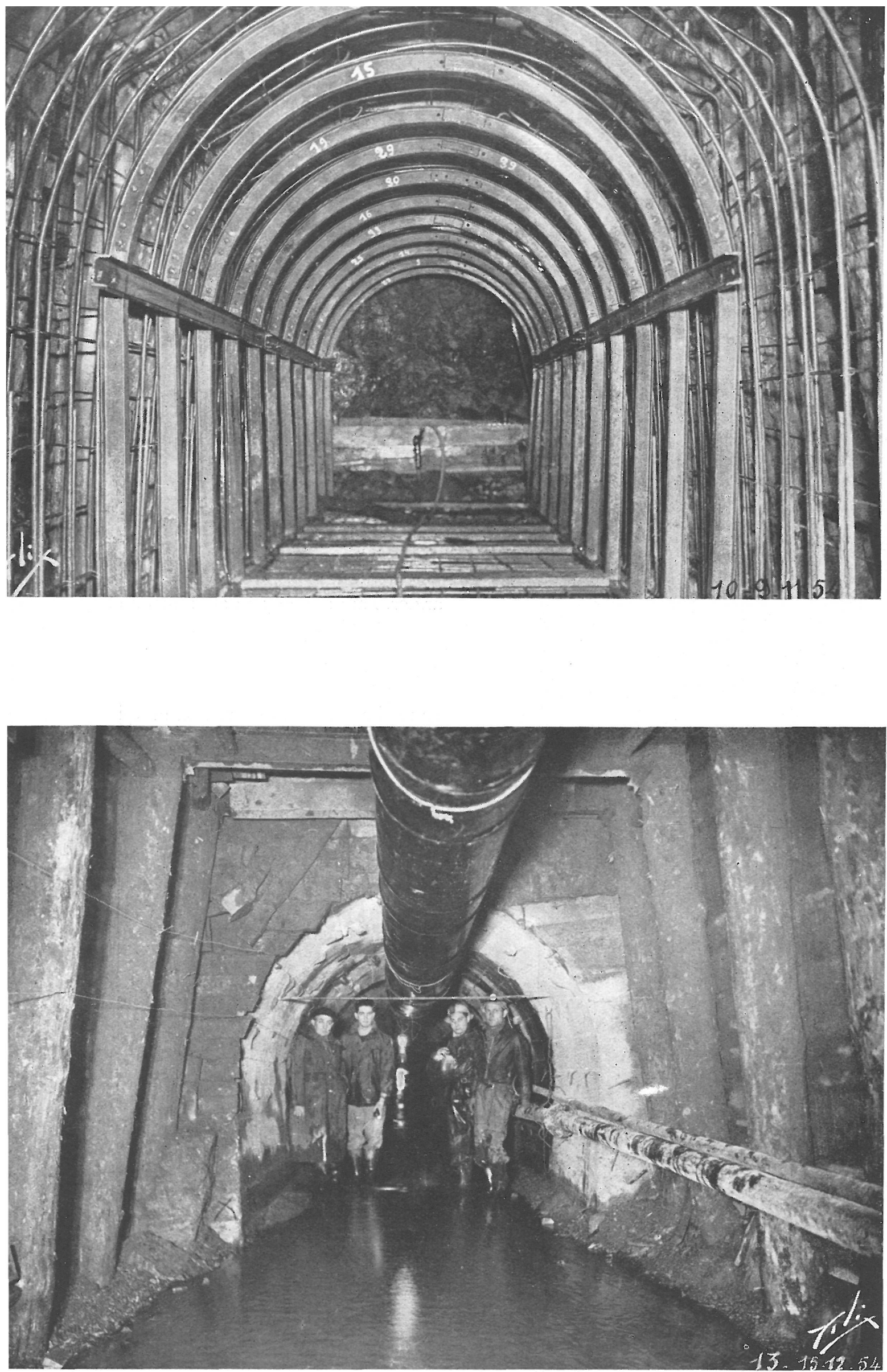

Un trozo de galería con las armaduras colocadas, en es-
Parte de galeria hormigonada para mayor seguridad entibación con cuadros de madera y aspecto del volu.
men de agua de infiltración.

I NFO R M ES DE LA C O N S T R U C C I O N 93 